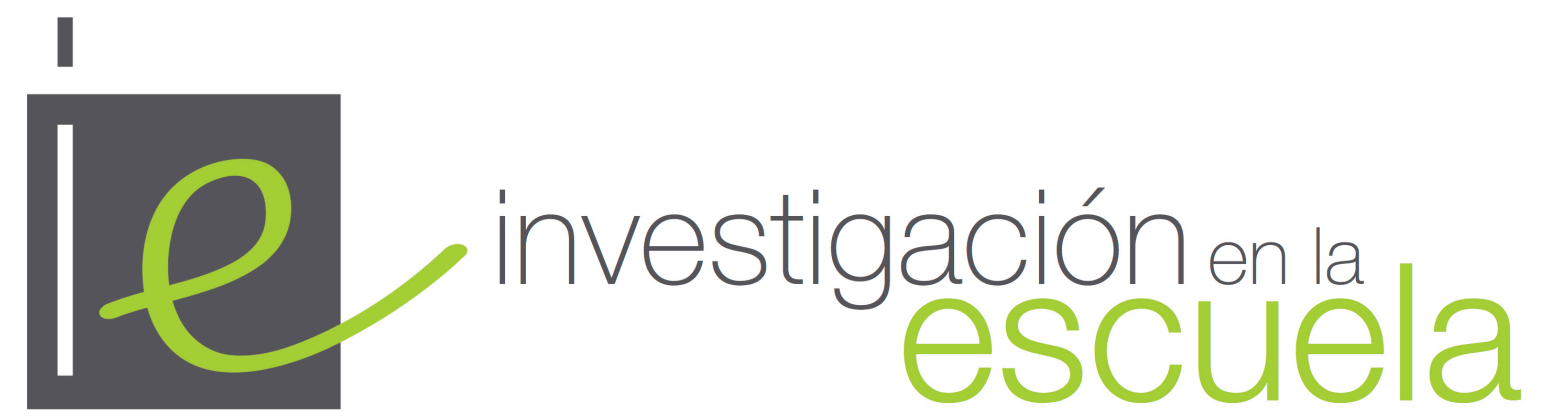

Revista internacional de investigación e innovación educativa

\title{
Dimensiones competenciales de la prevención y el afrontamiento de riesgos en Educación Primaria
}

\author{
M. Inés Gabari Gambarte y Raquel Sáenz. Mendía \\ Universidad Pública de Navarra \\ España
}

Citación: Gabari, M. I. y Sáenz, R. (2018). Dimensiones competenciales de la prevención y el afrontamiento de riesgos en Educación Primaria. Investigación en la Escuela, 94, 47-62. Recuperado de: http://www.investigacionenlaescuela.es/articulos/R94/R94-4

Resumen: Se pretende identificar los componentes de prevención y afrontamiento de riesgos escolares del alumnado de 8-9 años $(n=584)$ de Navarra (España), en relación con las competencias implicadas para la construcción de un entorno escolar más seguro. La recogida de datos se realiza mediante Cuestionario de Percepción de Accidentes Escolares (CPAE) y análisis de componentes principales, según método de rotación normalización Varimax con Kaiser. Se descubren cinco componentes en relación con las competencias: $1^{\circ}$ conductas seguras, promotoras de salud y bienestar; $2^{\circ}$ pensamiento reflexivo e introspectivo; $3^{\circ}$ representación e interpretación; $4^{\circ}$ estrategias preventivas y $5^{\circ}$ resolución de conflictos y educación en valores. Como resultado encontramos que el niño, en tanto que activo de salud, contribuye a la creación de entornos escolares más seguros. Palabras clave: "Competencia"; "accidente"; "salud escolar"; "grupos vulnerables"; "promoción de la salud".

\section{Competence dimensions of prevention and coping with risk in Elementary School}

Abstract: The aim is to identify the components of prevention and coping with school risks for 8-9 year-old students $(\mathrm{n}=584)$ of Navarra (Spain), in relation to the skills involved in the construction of a safer school environment. The data collection is done through the School Accident Perception Questionnaire (CPAE) and analysis of the main components, according to the Varimax rotation 
method with Kaiser. Five components are discovered in relation to the competences: 1st safe conducts, promoters of health and well-being; 2nd reflective and introspective thinking; $3 \mathrm{rd}$ representation and interpretation; 4th preventive strategies and 5th resolution of conflicts and education in values. As a result, we found that the child, as health asset, contributes to the creation of safer school environments.

Key words: "Competence"; "accident”; "school health"; "risk groups"; "health promotion”.

Les dimensions de la compétence de la prévention et de l'adaptation au risque dans l'École élémentaire

Resumè: L'objectif est d'identifier les composantes de prévention et de gestion des risques scolaires pour les élèves de 8 à 9 ans $(n=584)$ de Navarre (Espagne), en relation avec les compétences nécessaires à la construction d'un environnement scolaire plus sûr. La collecte des données se fait à travers le questionnaire de perception des accidents de l'école (CPAE), et l'analyse des principaux composants, selon la méthode de rotation Varimax avec Kaiser. Cinq composantes sont découvertes en relation avec les compétences: 1re conduite sécuritaire, promoteur de la santé et du bien-être; 2ème réflexion et réflexion introspective; 3e représentation et interprétation; 4ème stratégies préventives et 5 ème résolution des conflits et éducation aux valeurs. En conséquence, nous avons constaté que l'enfant, en tant qu'actif de santé, contribue à la création d'environnements scolaires plus sûrs.

Mots-clés: "Compétence"; "accident santé scolaire"; "groupes vulnérables"; "promotion de la santé".

\section{Introducción}

Expertos en Salud Comunitaria afirman que la capacidad que tiene el entorno para incidir en la salud de los ciudadanos es más importante que el código genético (Botello, Palacio, García, Margolles, Fernández, Hernán y Cofiño, 2013). En distintos barrios de una misma ciudad, la esperanza de vida de sus habitantes puede variar en seis años. Desde un punto de vista científico, es mucho más eficiente modificar un entorno que actuar sobre factores de riesgo en consultas. Ahí radica la importancia de incidir no sólo sobre los procesos biológicos, sino también sobre el trabajo, el lugar de estudio o laboral. Al centro de salud se unen las asociaciones de vecinos, los movimientos sociales y las instituciones educativas, entre otras entidades comprometidas con la salud de la comunidad.

En este sentido, la salud y la educación están íntimamente ligadas. El compromiso de la escuela para el desarrollo integral del individuo hace de la Educación para la Salud una necesidad educativa básica y un prerrequisito para adquirir las habilidades para la vida.

En los últimos veinte años, la comprensión de la realidad y las relaciones entre las personas y las distintas disciplinas han experimentado un cambio sustancial. Inmerso en este entramado resurge el término competencia. Tal y como mantienen autores varios al referirse a los desafíos para los programas de formación en salud pública (Cardona y Franco, 2005; Garzón, 2013; Gofín y Gofín, 2007; Leiva, George, Squicciarini, Simonsohn y Guzmán, 2015), la práctica educativa en la educación por competencias implica “...integrar metodología didáctica centrada en el aprendizaje, en la solución de problemas, estrechar la relación entre teoría y práctica" (Magaña, 2007, p. 369)

En esta línea, el Dominio 3: Promoción de la salud y participación social del Marco Regional de competencias en salud pública de la organización Panamericana de la Salud señala, entre otras acciones, la de "Analizar las relaciones de complementariedad entre la educación para la salud y la 
alfabetización en salud para establecer mejores prácticas de promoción de la salud" (Suárez et al., 2013).

Como referente de investigación en España encontramos el Proyecto "Competencias a adquirir por los jóvenes y el profesorado en educación para la salud durante la escolarización obligatoria" llevado a cabo por equipo interuniversitario de investigación COMSAL en el que participan las universidades de Valencia, Alicante, Elche, País Vasco, entre otras (Gavidia y Talavera, 2012).

Las características esenciales comunes a las competencias señaladas por Roegiers (2008) son: la movilización de un conjunto de recursos, la presencia de una finalidad, la relación a una familia de situaciones, el carácter disciplinar e interdisciplinar y la evaluabilidad. Estos rasgos son extensibles a las acciones promotoras de salud que se plantean en la infancia. En la Figura 1 se plasman las nueve subcompetencias de Educación para la salud incluidas en el currículo oficial de España para la Educación Primaria, conectadas con los Pilares del aprendizaje permanente propuestos por Delors en 1996.
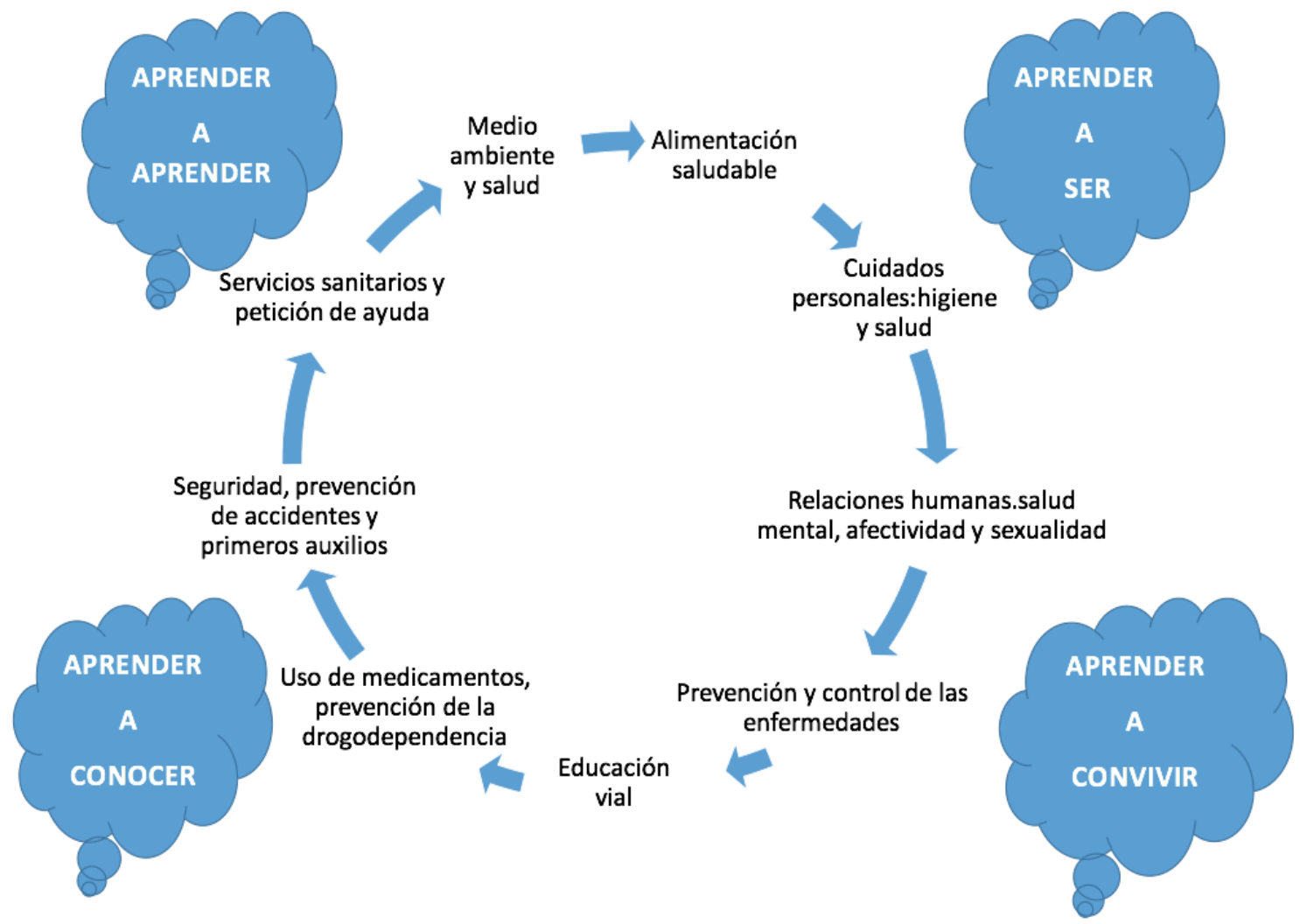

Figura 1. Subcompetencias EpS Modelo competencial del aprendizaje permanente. Fuente: Gabari y Sáenz (en Gavidia, 2016)

En relación con la subcompetencia "Seguridad, prevención de accidentes y primeros auxilios", los contextos escolares seguros facilitan la ausencia de daños causados tanto a alumnado como a docentes. Publicaciones recientes sobre legislación educativa (De Waal \& Grösser, 2014) amplían el ámbito de seguridad y protección, incluyendo en esta área problemas de falta de seguridad emocional (acoso) y falta de seguridad pedagógica (enseñanza discriminatoria). En concreto, en 
relación con el desarrollo de programas en el ámbito de la salud mental escolar algunos autores reconocen que:

"Las intervenciones promocionales y preventivas en el contexto escolar tienen efectos protectores en sus participantes. A nivel individual, estas intervenciones contribuyen a la disminución de problemas internalizantes y externalizantes y a fortalecer el desempeño académico. Además, generan efectos favorables en el ámbito de la familia y de la comunidad escolar, y son particularmente útiles cuando se dirigen a poblaciones con mayores riesgos a través de sistemas integrales y con programas multicomponentes, combinando esfuerzos del aula, la escuela y de toda la comunidad" (Leiva, George, Squicciarini, Simonsohn y Guzmán, 2015, p. 16).

Gairín (2015, p. 72), recoge la opinión de St. Leger, Young, Blanchard y Perry (2010) cuando afirman que "Seguridad y educación confluyen como dos concepciones inseparables", a lo que añade que no tiene sentido plantear una actividad educativa sin garantizar un marco adecuado de seguridad que la sustente. En definitiva, "Trabajar y estudiar en un ambiente seguro y saludable puede facilitar la tarea y mejorar el rendimiento, así como el bienestar y el confort de sus ocupantes" (Gairín, 2015, p. 72).

Las evidencias de algunos estudios (Gairín, 2011; Gairín \& Castro, 2011; Gairín, DíazVicario, Rosales y Sentillena, 2014) ponen de relieve que: 1) es preciso hablar de "seguridad integral", es decir de la integración de factores estructurales, de funcionamiento y de condiciones sociales derivadas de las relaciones (que es dónde se dan los casos de acoso y ciberacoso, entre otros); y 2) es imprescindible fomentar "procesos preventivos" en la cultura de la escuela, en sus valores y prácticas, en las competencias que fomenta.

Es en este marco en el que surge el interés por el estudio, lo que nos motiva a plantearnos el objetivo de identificar los componentes de prevención y afrontamiento de riesgos escolares expresados a través de cuestionario, por el alumnado de 8-9 años de la Comunidad Autónoma de Navarra (España), en relación con las competencias implicadas para la construcción de un entorno escolar más seguro.

\section{Método}

El diseño elegido es un estudio transversal de carácter cuantitativo, que permite describir comportamientos de las variables mediante el coeficiente de correlación de Pearson, cuyos valores absolutos oscilan entre 0 y 1 . La magnitud de la relación viene especificada por el valor numérico del coeficiente. $0 \leq r_{x y} \leq 1$.

En estudios epidemiológicos previos, como el realizado por el Equipo de investigación Educación y Salud de la Universidad Pública de Navarra (España) se evidencia que las edades de mayor vulnerabilidad comprenden tres períodos: $1^{\circ}$ ) de 12 a 24 meses, $2^{\circ}$ ) entre los cinco y seis años y $3^{\circ}$ ) a partir de los 9 años (Gabari, Sáenz, Idoate \& García, 2014).

La selección de los sujetos participantes se realiza en este último grupo. Para el cálculo del tamaño muestral se tiene en cuenta la fórmula de la distribución muestral de la media (Morales, 2012), resultando una $n=584$ niños y niñas de 8-9 años, representativa de una población de $\mathrm{N}=12.700$ escolares. El nivel de confianza elegido es de un $95 \%$, con un $\alpha=0,05$ y $z=1,96$.

Para la recolección de los datos se emplea un Cuestionario de Percepción de Accidentes Escolares (CPAE) validado (Gabari, Sáenz \& García, 2014). La primera parte consta de tres preguntas abiertas, que recogen información sobre las variables: 1) Incidencia diferencial de siniestralidad escolar según sexo, 2) Accidentes escolares evitables, 3) Mecanismos preventivos. Para evaluar la estructura del cuestionario a partir de las puntuaciones de las respuestas que lo componen, 
se ha llevado a cabo un análisis factorial exploratorio de las diferentes atribuciones, medidas de prevención y opinión respecto a los accidentes escolares, valoradas en el CPAE. La segunda parte, mediante la técnica de dibujo libre, solicita la representación plástica de los escolares respeto a la interpretación de la siniestralidad en el entorno escolar y su prevención.

El procedimiento que se sigue es el envío de una invitación por correo postal a los centros escolares de Navarra (España) a participar en el proyecto. Se confirma la aceptación mediante envío de un correo electrónico por parte de los equipos directivos y se concierta una sesión presencial del grupo de investigación interdisciplinar conformado por profesorado universitario de las Áreas de Enfermería y Pedagogía. Una vez presentado el estudio a los claustros respectivos, la recogida de datos tiene lugar en el contexto natural del aula, de manera integrada, como actividad curricular dirigida por el tutor y un miembro del equipo de investigación, con una duración de 60 minutos. Siguiendo el código de Helsinki, se ha resguardado la intimidad del alumnado y los centros educativos que participan en la investigación, así como la confidencialidad de su información personal para reducir al mínimo las consecuencias de la investigación sobre su integridad física, mental y social.

En cuanto al análisis, a partir de la recodificación de variables, se procede al cálculo de validez y fiabilidad ( $\alpha$ de Cronbach $=0,9)$ y del coeficiente de correlación de Pearson $(r \geq 0,5)$ y análisis de componentes principales, método de rotación normalización Varimax con Kaiser, con el programa SPSS v.23, adoptando un nivel de significación de $\mathrm{p}<0,05$.

\section{Resultados}

Los resultados obtenidos identifican los factores más importantes percibidos por los niños y niñas respecto a la prevención y afrontamiento de riesgos en el entorno escolar.

Mediante la técnica de Análisis de Componentes Principales (ACP) se calcula la validez estructural del cuestionario. Se puede comprobar la adecuación muestral ya que el estadístico de $\mathrm{KMO}$, con un valor de 0,823 (para Chi-cuadrado $=3315,223$ ), nos indica la proporción de la varianza que tienen en común las variables analizadas. Es un coeficiente cercano a la unidad que indicaría una perfecta adecuación de los datos a un modelo de análisis factorial. Mediante la prueba de esfericidad de Bartlett $(\mathrm{gl}=496)$, comprobamos que la significación es perfecta $(0,000)$ considerando idóneo el ajuste de las variables mediante el análisis factorial.

Una vez introducidas todas las variables, los datos obtenidos nos ofrecen una matriz definida positiva, condición necesaria para poder continuar con la metodología ACP. Las comunalidades obtenidas para cada una de las variables se recogen en la Tabla 1. Se identifican siete variables que explican la varianza: $1^{\mathrm{a}}$ ) La percepción de Mayor incidencia en niños que en niñas (,091); $2^{\mathrm{a}}$ ) La identificación de las causas de esta mayor siniestralidad en varones, la que recoge mayor parte de la varianza es la Causa 4: "Tipo de relación con iguales" (,906); $3^{a}$ ) Actitud proactiva de evitación (,368); $\left.4^{\mathrm{a}}\right)$ La Identificación de riesgos del entorno escolar, el que recoge mayor parte de la varianza es el Riesgo 3: "Manifestación de necesidad de ayuda" (,895); 5) La identificación de conductas proactivas de prevención, la que recoge mayor parte de la varianza es la Conducta 8: "Emplear recursos de ayuda con los iguales" (,850); $6^{a}$ ) La identificación de edad de más riesgo (,043); $7^{\mathrm{a}}$ ) La identificación del mecanismo de implicado en los accidentes escolares, el que recoge mayor parte de la varianza es el Mecanismo 4: "Elementos materiales implicados" (,654) y 8a) La elaboración de mensajes de prevención (,115). 
Tabla 1

Riesgos escolares percibidos por alumnado de 8-9 años de Navarra (España), curso 2012-2013

COMUNALIDAD DE VARIABLES

1. Percepción de mayor incidencia en niños que en niñas Inicial Extracción

2. Identificación de Causa de mayor siniestralidad en varones

$1,0,091$

3. Causa 1: Caraterística personal

$1,0,609$

4. Causa 2: Conducta de riesgo

$1,0,092$

5. Causa 3: Resultado del accidente

$1,0 \quad, 492$

6. Causa 4: Tipo de relación con iguales

$1,0 \quad, 649$

7. Actitud proactiva de evitación

$1,0,906$

8. Identificación Riesgos entorno escolares

$1,0 \quad 368$

9. Riesgo 1: Circunstancias fortuitas del accidente

$1,0,604$

10. Riesgo 2: Normas de Convivencia

$1,0 \quad, 538$

11. Riesgo 3: Manifestación de necesidad de ayuda

$1,0 \quad, 733$

12. Riesgo 4: Otros

$1,0 \quad, 895$

13. Identificación conductas proactivas de prevención

$1,0 \quad, 557$

14. Conducta 1: Ejercitar estrategias de anticipación

$1,0 \quad, 554$

15. Conducta 2: Respetar señales de aviso

$1,0 \quad, 500$

16. Conducta 3: Evitar contextos de Riesgo

$1,0 \quad, 434$

17. Conducta 4: Demandar ayuda al adulto

$1,0 \quad, 377$

18. Conducta 5: Mostrar conductas de convivencia positiva

$1,0,614$

19. Conducta 6: Rechazar conductas de convivencia negativa

$1,0 \quad, 441$

20. Conducta 7: Restituir daños ocasionados a un igual

$1,0 \quad, 371$

21. Conducta 8: Emplear recursos de ayuda con los iguales

$1,0 \quad, 775$

22. Conducta 9: Evaluar contextos y situaciones de riesgo

$1,0 \quad, 850$

$1,0 \quad, 531$

23. Conducta 10: Incorporar valores al estilo de vida

$1,0 \quad, 361$

24. Identificación Edad más riesgo

$1,0 \quad, 043$

25. Identificación mecanismo de accidentes escolares

$1,0 \quad 275$

26. Mecanismo 1: tipo de lesión

$1,0 \quad, 500$

27. Mecanismo 2: dinámica del accidente

$1,0 \quad, 591$

28. Mecanismo 3: personas presentes

$1,0 \quad, 437$

29. Mecanismo 4: elementos materiales implicados

$1,0 \quad 654$

30. Mecanismo 5: acciones realizadas

$1,0,606$

31. Mecanismo 6: señal de aviso sugerida

$1,0 \quad, 136$ 
32. Identificación de mensaje de prevención (eslogan)

$1,0 \quad, 115$

En la Tabla 2 se recoge la matriz factorial de elección de los componentes principales de la muestra, realizada a partir de una matriz de correlaciones. Desde el componente número cinco el autovalor se aproxima a la unidad y además el porcentaje de la varianza explicada acumulada asciende al 49,064\%, por lo que se estima que cinco es un número de factores suficientes. Para conseguir una interpretación clara de los datos se ha realizado el procedimiento de Rotación Factorial (Método de extracción: Análisis de los componentes principales y Método de rotación: Normalización Varimax con Kaixer).

Tabla 2

Varianza total explicada de riesgos escolares/afrontamiento percibidos por alumnado de 8-9 años de Navarra (España), curso 2102-2013. Método de extracción. Análisis de componentes principales

Sumas de las

saturaciones al

Componente cuadrado de la Suma de las saturaciones al cuadrado de la rotación extracción

\begin{tabular}{lllll}
\hline & $\%$ acumulado & & $\%$ varianza & $\%$ acumulado \\
\hline 1 & 23,511 & 6,924 & 21,639 & 21,639 \\
2 & 32,545 & 2,756 & 8,612 & 30,251 \\
3 & 39,239 & 2,462 & 7,694 & 37,945 \\
4 & 44,392 & 1,861 & 5,816 & 43,761 \\
5 & 49,064 & 1,697 & 5,303 & 49,064
\end{tabular}


Se agrupan los componentes junto con sus atributos, mostrando los valores situados por encima de 0,5 y el porcentaje total de la varianza que recoge cada uno de ellos (ver Tabla 3). Existen correlaciones positivas y alguna negativa; la máxima puntuación $(0,893)$ la alcanzan los dos últimos componentes (C4 y C5).

Tabla 3

Correlación lineal de los componentes identificados de riesgos escolares/afrontamiento percibidos por alumnado de 8-9 años de Navarra (España), curso 2102-2013

\begin{tabular}{llllll}
\hline Componente & C1 & C2 & C3 & C4 & C5 \\
\hline C1 &, 936 &, 291 &,- 088 &, 172 &, 040 \\
C2 &, 294 &,- 625 &, 680 &,- 154 &,- 190 \\
C3 &,- 188 &, 555 &, 647 &, 456 &,- 172 \\
C4 &,- 026 &,- 210 &, 151 &, 367 &, 893 \\
C5 &, 026 &, 415 &, 297 &,- 777 &, 367 \\
\hline
\end{tabular}

C: componente

Por último, se renombran los componentes en relación con las Competencias de aprendizaje a lo largo de la vida (lifelong learning), evidenciándose que la dimensión competencial precisa de una combinación de conocimientos, destrezas y actitudes necesarias para desarrollar estilos de vida saludables, que incorporen destrezas de prevención y comportamientos de seguridad en el entorno escolar. En la Tabla 4 se detallan los cinco componentes.

Tabla 4

Combinación competencial de conocimientos, destrezas y actitudes de prevención de accidentes en el entorno escolar, percibida por alumnado de 8-9 años de Navarra (España), curso 2012-2013

Componente 1

Porcentaje

de varianza

total

CONDUCTAS SEGURAS, PROMOTORAS DE SALUD Y

explicada

BIENESTAR

\section{COMPETENCIAS DE SALUD Y SEGURIDAD}

P.4. Causa 2: Conducta de riesgo $\quad 0,620$

P.5. Causa 3: Resultado de accidente $\quad 0,777$

P.6. Causa 4: Tipo de relación con iguales $\quad 0,922$

P.11. Riesgo 3: Manifestación de necesidad de ayuda $\quad 0,913$

P.15. Conducta 2: Respetar señales de aviso $\quad 0,631$

P.17. Conducta 4: Demandar ayuda al adulto $\quad 0,775$

P.18. Conducta 5: Mostrar conductas de convivencia positiva $\quad 0,632$

P.19. Conducta 6: Rechazar conductas de convivencia negativa $\quad 0,584$

P.20. Conducta 7: Restituir daños ocasionados a un igual $\quad 0,873$ 
P.21. Conducta 8: Emplear recursos de ayuda con los iguales $\quad 0,881$

P.22. Conducta 9: Evaluar contextos y situaciones de riesgo

Componente 2

PENSAMIENTO REFLEXIVO E INTROSPECCIÓN

COMPETENCIAS SOCIALES Y CÍVICAS

P.2. Identificación de Causa de mayor siniestralidad en $\quad 0,721$

$\begin{array}{ll}\text { varones } & 0,574\end{array}$

P.8. Identificación Riesgos entorno escolares $\quad 0,616$

P.10. Riesgo 2: Normas de Convivencia $\quad 0,626$

P.12. Riesgo 4: Evitar otros accidentes $\quad 0,529$

P.13. Identificación conductas proactivas de prevención

$\begin{array}{ll}\text { Componente } 3 & 6,693 \%\end{array}$

REPRESENTACIÓN E INTERPRETACIÓN

COMPETENCIAS

COMUNICACIÓN LINGÚISTICA

CONCIENCIA Y EXPRESIÓN CULTURAL

P.27. Mecanismo 2: dinámica del accidente $\quad 0,757$

P.29. Mecanismo 4: elementos materiales implicados $\quad 0,767$

P.30. Mecanismo 5: acciones realizadas $\quad 0,759$

Componente $4 \quad 5,154 \%$

ESTRATEGIAS PREVENTIVAS

COMPETENCIA APRENDER A APRENDER

$\begin{array}{lcc}\text { P.7. Actitud proactiva de evitación } & 0,558 \\ \text { P.9. Riesgo 1: Circunstancias fortuitas del accidente } & 0,610 & \\ \text { P.16. Conducta 3: Evitar contextos de Riesgo } & 0,541 & 4,672 \% \\ \text { Componente 5 } & & \\ \text { RESOLUCIÓN DE CONFLICTOS, EDUCACIÓN EN } & \\ \text { VALORES } & \\ \text { COMPETENCIAS EMOCIONALES } & 0,522 \\ \text { P.23. Conducta 10: Incorporar valores al estilo de vida } & 0,594 \\ \text { P.28. Mecanismo 3: personas presentes } & \end{array}$

\section{Discusión}

La aplicabilidad del modelo competencial desde las etapas tempranas permite que los alumnos y alumnas conozcan y perciban su salud, hagan del estilo de vida saludable un hábito permanente, aprendan a prevenir situaciones de riesgo y adopten conductas de bienestar, en espacios de convivencia en los que la salud física y mental se interioriza como un bien social.

Desde estas señas de identidad, el modelo de aprendizaje por competencias cobra sentido al considerar que el proceso de enseñanza-aprendizaje no se reduce únicamente al desarrollo del currículo explícito, pues hay otros ámbitos en los centros escolares en los que la práctica de la convivencia, las actividades complementarias y las actuaciones en el entorno ofrecen al alumnado escenarios reales de aprendizaje, como parte del currículo implícito u oculto.

En este sentido, se establece un paralelismo entre los componentes identificados en este trabajo mediante el análisis factorial exploratorio, y las competencias del Marco Europeo de Educación Permanente (2006), las cuales a su vez se sustentan en los 'pilares básicos de la Educación' propuestos por Delors (1996). 
En concreto, el Componente 1 conductas seguras, promotoras de salud y bienestar, muestra una visión infantil respecto a la siniestralidad escolar que traspasa el horizonte de la lesión en sí misma, su mecanismo, elementos implicados y sus consecuencias, para ampliar su mirada respecto al contexto educativo como espacio en el que se fomentan hábitos de salud y seguridad. Incluye las conductas de riesgo y los resultados de los accidentes como causas de los mismos. Las relaciones entre iguales, la regulación normativa del adulto, además de su actuación como guía o facilitador, aparece como propuesta de mejora que permite evitar estos sucesos. En conjunto, estas actitudes pueden desarrollar habilidades y conductas de afrontamiento que promuevan el autocuidado y la génesis de estilos de vida saludables. Se fortalece el rol formador y socializador de la escuela en relación a procesos vinculados a la salud como un todo: desarrollo psicosocial, competencias emocionales, responsabilidad colectiva etc., tal y como se recoge en diversas propuestas (Cummings, Norton \& Koepsell, 2001; Eichel \& Goldman, 2001; Gabari, Sáenz, Idoate \& García, 2014; Miller, Romano \& Spice, 2000; Ruíz, Soriano y Cabrera, 2010; Sáenz Mendía, Gabari Gambarte \& García García, 2016; Talavera y Gavidia, 2013).

Respecto al Componente 2, pensamiento reflexivo e introspectivo, agrupa a aquellas operaciones mentales que suscitan el cuestionamiento interno en torno a las causas que conducen a los accidentes. El desarrollo de la empatía favorece una conducta prosocial que puede reducir la agresividad, potenciando la autoeficacia y la responsabilidad personal y social de los escolares. Junto a los aspectos cognoscitivos del aprendizaje, otras dimensiones no menos importantes de la educación integral son el desarrollo de la personalidad, la socialización, la formación del pensamiento o la conciencia ético-moral a las que hacen referencia González, Ordóñez, Montoya y Gil (2014). El hecho de despertar la conciencia sobre sus acciones induce a nuevos modelos de afrontamiento de situaciones que entrañan peligros. Las utilizaciones de estas estrategias en la vida diaria permiten adoptar actitudes seguras para sí mismo y para los de su alrededor identificando activos de salud en su propio entorno que fortalecen el Sentido de Coherencia (SOC) propuesto por Lindström \& Erikson (2006).

El Componente 3, representación e interpretación, hace referencia a los elementos que tienen que ver con la comunicación lingüística, conciencia y expresión cultural. El proceso de simbolizar la realidad y la fantasía, los hechos objetivos y los subjetivos en un papel, permite contrastar las distintas percepciones que tienen de los acontecimientos de su alrededor. El desarrollo de la sensibilidad estética, el sentido de la observación, el tratamiento del color y de las imágenes deja un testimonio indeleble del entorno que recrean, como hemos constatado en las investigaciones en las que humanizan los espacios infantiles a través del arte (López-Bosch, Fernández-Añino y ÁvilaValdés, 2002; Macnaughton, White \& Stacy, 2005; Pont, 2014; Stern, 2008; Ullán y Belver, 2008).

En el Componente 4, las estrategias preventivas se vinculan con la competencia de aprender a aprender. Se evidencia la creencia de que los accidentes se pueden evitar, de una manera intuitiva, con un pensamiento poco estructurado, ya que a esta edad no es posible una comprensión total del complejo mecanismo de producción de los accidentes. Los hábitos de cautela y de precaución, no sólo a nivel individual, sino teniendo en cuenta la repercusión de las conductas individuales en el resto del grupo, objetivan que a la edad de ocho-nueve años, las personas son capaces de conectar con los demás e identificar nuestras emociones y las de las otras personas. Gracias a esa capacidad tan innatamente humana, somos capaces de sintonizar con la parte más íntima de quienes nos rodean y desarrollar estrategias de afrontamiento en el caso de la prevención de accidentes escolares o la adaptación de estilos de vida saludables a nivel individual y comunitario (Gabari y Sáenz, 2016; Gairín \& Castro, 2011; Mangrulkar, Whitman y Posner, 2001).

Por último, el Componente 5, Resolución de conflictos y la educación en valores, se muestran ligados a las competencias emocionales. La adopción de valores de carácter general propuestos por los propios escolares participantes suscita una vía de gran interés en el ámbito 
educativo, para mejorar el equilibrio mental y el desarrollo socioemocional del alumnado. La línea de investigación de la educación emocional se centra en establecer la utilidad de este constructo en diversas áreas de las personas, ya que ésta determina nuestro comportamiento e influye significativamente en la vida, en la manera de comprender, percibir y manejar de forma adaptativa las propias emociones, como se pone de manifiesto en numerosos estudios (Greenberg et al., 2003; Lessa et al., 2017).

Tal y como afirman Ríos-Cortázar, Gasca-García, Franco-Martínez y Tolentino-Mayo (2014) en su estudio sobre Narrativa infantil en entornos escolares como estrategia de promoción de la salud, los planes de promoción de salud en entornos escolares tendrían mayor profundidad si se estructurasen considerando la organización y la participación activa de los niños como su eje central, en concreto, desde nuestro estudio, contribuiría a disminuir las estadísticas de siniestralidad en el entorno educativo.

El alcance del estudio se dirige a contribuir a la consecución de objetivos, orientar temáticas y fortalecer mecanismos de colaboración interdisciplinar e interinstitucional, en las campañas promovidas desde los ámbitos de Salud Pública y de Educación, incorporando la visión preventiva de los propios escolares, dado que es una aportación escasamente visibilizada en estudios precedentes.

Las limitaciones que nos hemos encontrado comienzan por tener escasos referentes de estudios previos con los que comparar los hallazgos. Por otro lado, existe una dificultad real de trascender modelos de salud asistenciales, insertados en las culturas educativas que, si bien, en el discurso asumen la visión preventiva no acaban de arbitrar mecanismos en la praxis, que incorporen a la infancia como un activo de salud en presente y no sólo a futuro. Se pone de relieve que el profesorado tiene que ser consciente de los conocimientos previos sobre los contenidos a enseñar que tiene el alumnado, que pueden constituir alternativas al conocimiento a enseñar y que, podrían entrar en conflicto con la comprensión de los conceptos (Solís, Porlán, Martín del Pozo \& Harres, 2016). A modo de conclusión, respecto al logro del objetivo de identificar los componentes de prevención y afrontamiento de riesgos escolares, consideramos que nuestros hallazgos pueden servir de orientación para integrar, de manera transversal y a nivel competencial, estilos de vida saludables como clave en la construcción de entornos escolares más seguros.

En concreto, tal y como afirma Magaña (2007, p. 370) para el desarrollo de competencias, en este caso de prevención de riesgos y promoción de la salud, "urge reorientar la práctica pedagógica, lo cual implica integrar metodología didáctica centrada en el aprendizaje, en la solución de problemas, estrechar la relación entre teoría y práctica, y eso sólo se logra con herramientas pedagógicas como el análisis de casos, el aprendizaje basado en proyectos y el trabajo en colaboración", con el apoyo de recursos diversos entre los que cobra especial relevancia la simulación a través de soportes virtuales.

Desde este estudio apostamos por reivindicar la oportunidad de incorporar a la comunidad educativa la figura profesional de enfermería escolar. Según Casado (2015) esta figura está asentada en países como EE.UU. (más del 75\% de las escuelas la tienen), Reino Unido (ampliamente extendida), Francia (con la figura reglada de l'Infirmier de l'Éducation nationale), Escocia (con al menos una enfermera por centro educativo) o Suecia (en dónde cada enfermera atiende a más de un centro). En España, dado que dicha figura no está implantada en la enseñanza pública, estamos ante un momento de debate político, social y sanitario que esperamos apueste por su inclusión de manera generalizada. 


\section{Referencias}

Botello, B., Palacio, S., García, M., Margolles, M., Fernández, F., Hernán, M., y Cofiño, R. (2013). Metodología para el mapeo de activos de salud en una comunidad. Gaceta Sanitaria, 27, 180183. http://dx.doi.org/10.1016/j.gaceta.2012.05.006

Cardona, A., y Franco, A. (2005). La salud pública como disciplina científica: fundamento para los programas de formación académica. Revista Facultad Nacional de Salud Pública, 23 (2), 107-114

Casado, A. E. (2015). Enfermería Escolar, La Situación Hoy En Día. Revista Enfermería Cy L, 7 (1), $56-61$.

Cummings, P., Norton, R. \& Koepsell, T. (2001). Rates, rate denominators, and rate comparisons. In: F., Rivara, D.C., Grossman, P., Cummings, R.V., Maier, T.D., Koepsell (Eds.), Injury Control A Guide to Research and Program Evaluation, (pp. 64-74). Cambridge: Cambridge University Press.

De Waal, E. \& Grösser, M. (2014). On safety and security in education: Pedagogical needs and fundamental rights of learners. Educar, 50, 339-361. http://dx.doi.org/10.5565/rev/educar.44.

Delors, J. (1996). La educación encierra un tesoro. París: UNESCO.

Eichel, J. D. S., \& Goldman, L. (2001). Safety makes sense: a program to prevent unintentional injuries in New York City public schools. Journal of School Health, 71 (5), 180-183. http://dx.doi.org/10.1111/j.1746_1561.2001.tb07312.x.

Gabari, M. I. y Sáenz, R. (2016). La prevención de accidentes escolares desde el desarrollo de competencias. En V. Gavidia, Los ocho ámbitos de la EPS en la escuela (pp. 331-356). Valencia: Tirant lo Blanch.

Gabari, M. I., Sáenz, R. \& García, J. M. (2014). Children's Drawings: experiences of children in accident prevention and health promotion. WER A Focal Meeting, Edinburgo, 19-21. Disponible en: http://www.sera.ac.uk/documents/2014/SERA_2014_Book_of_abstracts.pdf.

Gabari, M. I., Sáenz, R., Idoate, V. \& García, J. M. (2014). Assessing Segurikaschool: an injury prevention multimedia tool for schools. Procedia-Social and Behavioral Sciences, 132, 654-660. http://dx.doi.org/10.1016/j.sbspro.2014.04.368.

Gairín, J. (2015). Promover y gestionar el conocimiento colectivo para mejorar la cultura y la práctica de la seguridad en educación. Cuadernos de estrategia, (172), 59-84.

Gairín, J. (Coord.). (2011). Qüestionari d'autoavaluació de la seguretat integral per a centres educatius. Revista Catalana de Seguretat Pública, 24, 193-212.

Gairín, J. \& Castro, D. (2011). Safety in schools: An integral approach. International Journal of Leadership in Education, 2011, 14 (4), 457-474. Disponible en: http://dx.doi.org/10.1080/136031. 24.2011.585664.

Gairín, J., Díaz-Vicario, A., Rosales, M. y Sentillena, X. (2014). La autoevaluación para la mejora de la seguridad integral en los centros educativos. Educar 50/2, 2014, 363-381.

Garzón, A. (2013). Transformaciones y nuevas perspectivas profesionales y académicas de la Psicología de la Salud. Revista Latinoamericana de Psicología, 45 (2), 241-252. Disponible en: http:/ / www.redalyc.org/articulo.oa?id=80528401006.

Gavidia, V. y Talavera, M. (2012). La construcción del concepto de salud. Didáctica de las ciencias experimentales y sociales, 26, 161-175. http://dx.doi.org/10.7203/dces.26.1935.

Gofín, J., y Gofin, R. (2007). Atención primaria orientada a la comunidad: un modelo de salud pública en la atención primaria. Rev Panam Salud Publica/Pan Am J Public Health, 21 (2/3), 177-185.

González, R., Ordoñez, A., Montoya, I. y Gil, J. (2014). Intervención en habilidades sociales para Educación Primaria. International Journal of Developmental and Educational Psychology, 600-606. 
Greenberg, M. T., Weissberg, R. P., O'Brien, M. U., Zins, J. E., Fredericks, L., Resnik, H. \& Elias, M. J. (2003). Enhancing school-based prevention and youth development through coordinated social, emotional, and academic learning. American psychologist, 58, 466-475. http://dx.doi.org/10.1037/0003-066X.58.6-7.466.

Leiva, L., George, M., Squicciarini, A. M., Simonsohn, A., y Guzmán, J. (2015). Intervención preventiva de salud mental escolar en adolescentes: desafíos para un programa público en comunidades educativas. Universitas Psychologica, 14 (4), 14-26.

Lessa, R., Scattolin, C., Oliveira, R., Lessa, B., Oliveira-Campos, M., Ratzsch de Andreazzi, M.A. \& Carvalho, D. (2017). Health promotion in school environment in Brazil. Rev. Saúde Pública, 51. http://dx.doi.org/10.1590/s1518-8787.2017051006709.

Lindström, B. \& Eriksson, M. (2006). Contextualizing salutogenesis and Antonovsky in public health development. Health promotion international, 21, 238-244. https://doi.org/10.1093/heapro/dal016.

López-Bosch, M. A., Fernández-Añino, M. y Ávila-Valdés, N. (2002). La representación de lo bueno y lo malo en el dibujo infantil: un estudio iconográfico. Arte, Individuo y Sociedad, 195-203.

Macnaughton, J., White, M. \& Stacy, R. (2005). Researching the benefits of arts in health. Health Education, 105, 332-339.

Magaña, L. (2007). La educación virtual en la formación gerencial de cuadros directivos. Salud Pública de México, 49, 368-371.

Mangrulkar, L., Whitman, C. V. y Posner, M. (2001). Enfoque de habilidades para la vida para un desarrollo saludable de niños y adolescentes. México: Organización Panamericana de la Salud.

Miller, T., Romano, E. \& Spice, R. S. (2000). The teaching of the safety and health in the educational centers. Future of children, 10, 1-15.

Morales, P. (2012). Tamaño necesario de la muestra: ¿Cuántos sujetos necesitamos? Estadística aplicada a las Ciencias Sociales, 24, 1-24.

Pont, T. (2014). Los test gráficos en el psicodiagnóstico. Síntesis: Madrid.

Ríos-Cortázar, V., Gasca-García, A., Franco-Martínez, M. \& Tolentino-Mayo, L. (2014). Narrativa infantil en entornos escolares: Una estrategia de promoción de la salud. Salud Pública de Méx, $56,130-138$.

Roegiers, X. (2008). Las reformas curriculares guían a las escuelas: pero ¿hacia dónde? Profesorado. Revista de curriculum y formación del profesorado, 12 (3), 1-36. Disponible en: http://www.ugr.es/local/recfpro/rev123ART4.pdf.

Ruíz, B., Soriano, M. y Cabrera, A. (2010). Prevención de la accidentalidad infantil en Andalucía: aprender a crecer con seguridad. Anales de Pediatría, 73, 249-256. https://doi.org/10.1016/j.anpedi.2010.03.009.

Sáenz Mendía, R., Gabari Gambarte, M. I., y García García, J. M. (2016). Recursos de salud positiva: estudio exploratorio con grupos del ámbito escolar de Pamplona. Atención Primaria, 48 (2), 140-141. https://doi.org/10.1016/j.aprim.2015.06.003.

Solís, E., Porlán, R., Martín del Pozo, R. \& Harres, J. (2016). Aprender a detectar las ideas del alumnado de Primaria sobre los contenidos escolares de ciencias. Investigación en la escuela, 88, 46-62. Disponible en: www.investigacionenlaescuela.es/articulos/R88/R88-3.

St Leger, l., Young, I, Blancharrd, C. y Perry, M. (2010). Promover la salud en la escuela: de la evidencia a la acción. Copenhague: UIPES, 2010. Disponible en: http://www.iuhpe.org/uploaded/Activities/Scientific_Affairs/CDC/PSeE_deEvidenciaala Accion_ES_WEB.pdf.

Stern, A. (2008). Del dibujo infantil a la semiología de la expresión. Valencia: Carena. 
Suárez, J., Godue, C., García, J. F., Magaña, L., Rabionet, S., Concha, J., Vázquez, M., Darío, R., Mújica, O., Cabezas, C., Liendo, L. y Castellanos, J. (2013). Competencias esenciales en salud pública: un marco regional para las Américas. Rev. Panam Salud Pública, 34, 47-53.

Talavera, M. y Gavidia, V. (2013). Percepción de la educación para la salud en el personal docente y el sanitario. Didáctica de las Ciencias Experimentales y Sociales, 27, 115-129. http://doi.org/10.7203/dces.27.2569.

Ullán, A. y Belver, M. H. (2008). Cuando los pacientes son niños. Humanización y calidad en la hospitalización pediátrica. Madrid: Envida.

\section{Información sobre los autores}

Autor: M. Inés Gabari Gambarte

Institución: Universidad Pública de Navarra

Email: igabari@unavarra.es

Información biográfica: Doctora en Ciencias de la Educación. Profesora Departamento Psicología y Pedagogía. Universidad Pública de Navarra. Edificio los Magnolios. Campus de Arrosadía, s/n 31004 Pamplona (Navarra, Spain).

Autor: Raquel Sáenz Mendía

Institución: Universidad Pública de Navarra

Email: raquel.saenz@unavarra.es

Información biográfica: Doctora en Ciencias de la Salud. Profesora Departamento de Ciencias de la Salud de la Universidad Pública de Navarra. Avenida de Barañáin, s/n. 31008 de Pamplona (Navarra, Spain). 


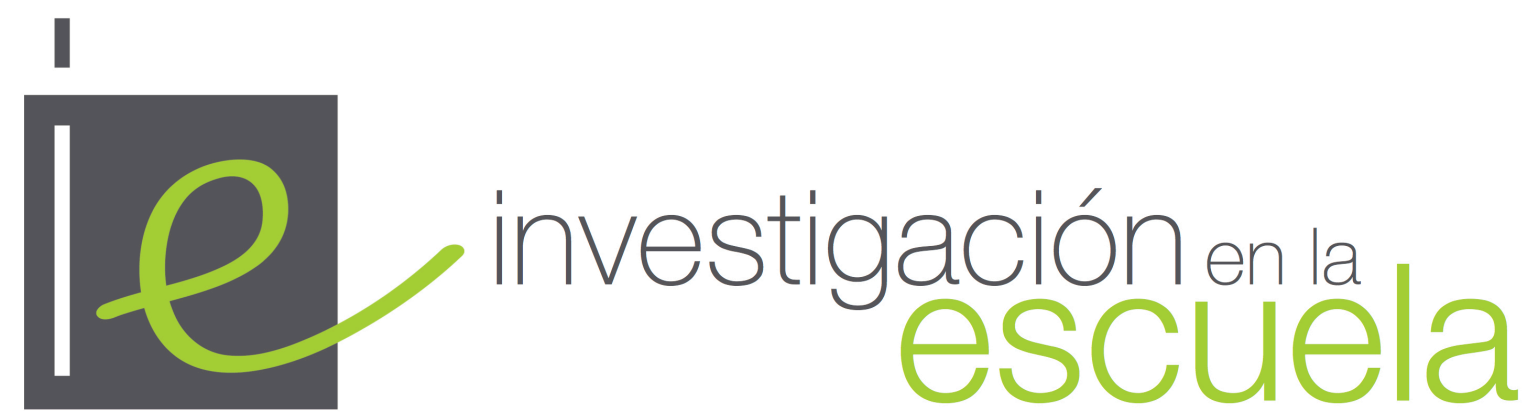

Revista académica evaluada por pares y de acceso abierto

Número 94

30 de abril de 2018

ISSN 2443-9991

\section{(c)}

SOMERIGHISRESERVELLOS/as lectores/as pueden copiar, mostrar, y distribuir este artículo, siempre y cuando se de crédito y atribución al autor/es y a Investigación en la Escuela, se distribuya con propósitos no-comerciales, no se altere o transforme el trabajo original. Más detalles de la licencia de CreativeCommons se encuentran en http://creativecommons.org/licenses/by-nc-sa/3.0 Cualquier otro uso debe ser aprobado en conjunto por el autor/es, o Investigación en la Escuela.

पis? investigacion-en-la-escuela

Contribuya con comentarios y sugerencias en la web de la revista. Por errores y sugerencias contacteasecretaria@investigacionenlaescuela.es 


\section{Investigación en la escuela}

Consejo de dirección: Ana Rivero García (Universidad de Sevilla), Nicolás de Alba Fernández (Universidad de Sevilla), Pedro Cañal de León (Universidad de Sevilla), Francisco F. García Pérez (Universidad de Sevilla), Gabriel Travé González (Universidad de Huelva), Francisco F. Pozuelos Estrada (Universidad de Huelva)

Dirección: Ana Rivero García y Nicolás de Alba Fernández

Secretaría de edición: Elisa Navarro Medina

\section{Consejo editorial}

José Félix Angulo Rasco. Universidad de Cádiz Rosa Má Ávila Ruiz. Universidad de Sevilla Pilar AzcárateGoded. Universidad de Cádiz Juan Bautista Martínez Rodríguez. Universidad de Granada

Nieves Blanco García. Universidad de Málaga Fernando Barragán Medero. Universidad de La Laguna José Carrillo Yáñez. Universidad de Huelva José Contreras Domingo. Universidad de Barcelona. Luis C. Contreras González. Universidad de Huelva Ana $\mathbf{M}^{\mathbf{a}}$ Criado García-Legaz. Universidad de Sevilla Rosario Cubero Pérez. Universidad de Sevilla José $\mathbf{M}^{\mathbf{a}}$ Cuenca López. Universidad de Huelva Jesús Estepa Giménez. Universidad de Huelva Rafael Feito Alonso. Universidad Complutense (Madrid)

Francisco José García Gallardo. Universidad de Huelva

Soledad García Gómez. Universidad de Sevilla J. Eduardo García Díaz. Universidad de Sevilla
Fernando Hernández Hernández. Universidad de Barcelona

Salvador Llinares Ciscar. Universidad de Alicante Alfonso Luque Lozano. Universidad de Sevilla Rosa Martín del Pozo. Universidad Complutense (Madrid)

José Martín Toscano. IES Fernando Herrera (Sevilla) Jaume Martínez Bonafé. Universidad de Valencia F. Javier Merchán Iglesias. Universidad de Sevilla Emilia Moreno Sánchez. Universidad de Huelva. Rosario Ortega Ruiz. Universidad de Córdoba Antonio de Pro Bueno. Universidad de Murcia Fco. de Paula Rodríguez Miranda. Universidad de Huelva

Pedro Sáenz-López Buñuel. Universidad de Huelva Antoni Santisteban Fernández. Universidad Autónoma (Barcelona)

Emilio Solís Ramírez. Catedrático de IES. $\mathbf{M}^{\mathbf{a}}$ Victoria Sánchez García. Universidad de Sevilla. Magdalena Suárez Ortega. Universidad de Sevilla

\section{Consejo asesor}

Manuel Área Moreira. Universidad de La Laguna

Jaume Carbonell. Director Cuadernos de Pedagogía. Barcelona

César Coll. Universidad de Barcelona

Christopher Day. Universidad de Nothingham. U.K.

Juan Delval. Universidad Nacional de Educación a Distancia

John Elliott. Universidad de East Anglia. Norwich. U.K.

José Gimeno Sacristán. Universidad de Valencia

André Giordan. Universidad de Paris VII y Ginebra

Francisco Imbernón. Universidad de Barcelona

Ángel Pérez Gómez. Universidad de Málaga

Rafael Porlán Ariza. Universidad de Sevilla

Francesco Tonucci. Instituto de Pedagogía del C.N.R. Roma

Jurjo Torres Santomé. Universidad de A Coruña 
